\title{
Representation of a trend in OFN during fuzzy observance of the water level from the crisis control center
}

\author{
Jacek M. Czerniak \\ Casimir the Great University in Bydgoszcz \\ Institute of Technology \\ ul. Chodkiewicza 30, 85-064 Bydgoszcz, Poland \\ Email: jczerniak@ukw.edu.pl
}

\author{
Wojciech T. Dobrosielski, Łukasz Apiecionek, Dawid Ewald \\ Casimir the Great University in Bydgoszcz, \\ Institute of Technology, \\ ul. Chodkiewicza 30, 85-064 Bydgoszcz, Poland \\ Email: \{wdobrosielski, lapiecionek, dawidewald \}@ukw.edu.pl
}

\begin{abstract}
This paper presents the issue of fuzzy arithmetic calculations in two different notations. The well-known L-R notation proposed by Dubois-Prade, which enjoys a well-earned recognition of the researchers dealing with fuzzy logic was presented on one hand. On the other hand, a OFN notation introduced by Kosiński was discussed. Comparative calculations were performed using the data of the benchmark "Dam and Crisis control center paradox". That benchmark is available in two versions, where the opposite trend is visible at the dam and at the CCC (Crisis control center). In one of the versions, the water level at the observed area decreases and increases at the dam, while in the other version the situation is opposite. The trend difference detection can aid the short-term forecast of the situation change at the monitored area. Results of the applied calculations in OFN notation show that this arithmetic is sensitive to trend differences related to the order characteristic for those numbers. Relationship between the fuzzy logic and the trend of the observed phenomena is an added value to the generalization of OFN and it is also a good signal for the future development of applications of such fuzzy calculations, being their unique feature at the same time.
\end{abstract}

\section{INTRODUCTION}

$\mathbf{T}$ HE HISTORY of artificial intelligence shows that new ideas were often inspired by natural phenomena. Many tourists come back with passion to beaches at the Oceanside and many sailors sail on tide water. The phenomenon of high and low tides, although well known, has been stimulating imagination and provoking reflexion on the perfection of the Creation for many ages. The casual observer in unable to precisely specify water level decline, but he or she can easily describe it using fuzzy concepts such as "less and less", "little" and "a bit". The same applies to increase of water in the observed basin. The observer can describe it such linguistic terms like "more", "lots of" or "very much". Such linguistic description of reality is characteristic to powerful and dynamically developing discipline of artificial intelligence like fuzzy logic. The author of Fuzzy logic is an American professor of the Columbia University in New York City and of Berkeley University in California - Lotfi A. Zadeh, who published the paper entitled "Fuzzy sets" in the journal "Information and Control" in 1965 [1]. He defined the term of a fuzzy set there, thanks to which imprecise data could be described using values from the interval $(0,1)$. The number assigned to them represents their degree of membership in this set. It is worth mentioning that in his theory L.Zadeh used the article on 3-valued logic published 45 years before by a Pole Jan Šukasiewicz [2]. That is why many scientists in the world regard this Pole as the "father" of fuzzy logic. Next decades saw rapid development of fuzzy logic. Another milestones of the history of that discipline should necessarily mention L-R representation of fuzzy numbers proposed by D.Dubois and H.Prade [3], [4], [5], which enjoys great successes today. Coming back to the original analogy, one can see some trend, i.e. general increase during rising tide or decrease during low tide, regardless of momentary fluctuations of the water surface level. This resembles a number of macro and micro-economic mechanisms where trends and time series can be observed. The most obvious example of that seem to be bull and bear market on stock exchanges, which indicate to the general trend, while shares of individual companies may temporarily fall or rise. The aim is to capture the environmental context of changes in economy or another limited part of reality. Changes in an object described using fuzzy logic seem to be thoroughly studied in many papers. But it is not necessarily the case as regards linking those changes with trend. Perhaps this might be the opportunity to apply generalization of fuzzy logic which are, in the opinion of authors of that concept, W.Kosiński [6] and his team, Ordered Fuzzy Numbers.

As the basis for experiments, let us assume the example of the dam and the impounding basin presented in the figure below (Fig. 1). Letter A indicates the water level measured during last evening measurement. Then there was a rapid surge of water in the night. Whereas morning measurement was marked using letter C. Measurements were imprecise to some extent due to rapid changes of weather conditions. It is also known that during the last measurement the safety valve $z 2$, was open and then the valve $z 1$ activated. The management of the dam faces the problem of reporting rapid surges of water to the disaster recovery centre. 


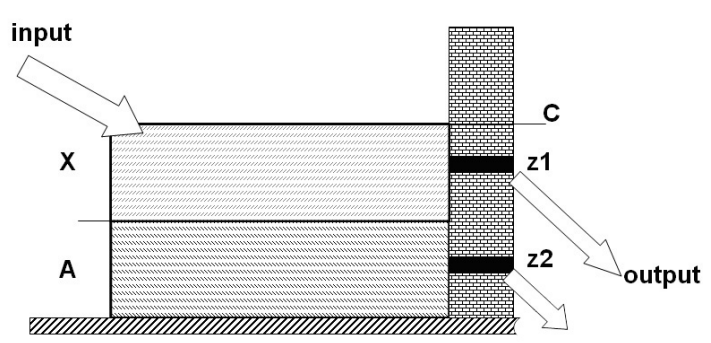

Fig. 1. The diagram of water flow in the impounding basin

\section{THEORETICAL BACKGROUND DESCRIPTION OF OFN}

\section{A. Some definitions of $O F N$}

Each operation on fuzzy numbers, regardless if it is addition, subtraction, division or multiplication, can increases the carrier value. Several operations performed on given L-R numbers can result in numbers that are too broad and as a result they can become less useful. Solving equations using conventional operations on fuzzy numbers [7] is usually impossible either. An $A+X=C$ equation can always be solved using conventional operations on fuzzy numbers only when $A$ is a real number. First attempts to redefine new operations on fuzzy numbers were undertaken at the beginning of the 1990-ties by Witold Kosiński and his PhD student P. SŞysz [8]. Further studies of W. Kosiński published in cooperation with P. Prokopowicz and D. Ślęzak [9], [7], [10] led to introduction of the ordered fuzzy numbers model OFN.

Definition 1: An ordered fuzzy number $A$ was identified with an ordered pair of continuous real functions defined on the interval $[0,1]$, i.e., $A=(f, g)$ with $f, g:[0,1] \longrightarrow R$ as continuous functions.

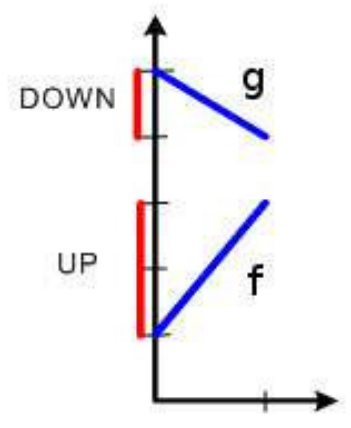

Fig. 2. Ordered fuzzy number

We call $\mathrm{f}$ and $\mathrm{g}$ the up and down-parts of the fuzzy number $A$, respectively. To be in agreement with the classical denotation of fuzzy sets (numbers), the independent variable of both functions $f$ and $g$ is denoted by $y$, and their values by $x$. [6]

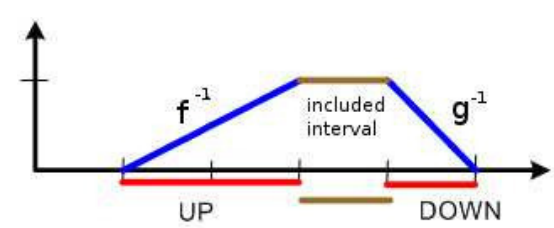

Fig. 3. OFN presented in a way referring to fuzzy numbers

Continuity of those two parts shows that their images are limited by specific intervals. They are named respectively: $U P$ and $D O W N$. The limits (real numbers) of those intervals were marked using the following symbols: $U P=\left(l_{A}, l_{A}^{-}\right)$ and $D O W N=\left(l_{A}^{+}, p_{A}\right)$. If both functions that are parts of the fuzzy number are strictly monotonic, then there are their inverse functions $x_{u p}^{-1}$ and $x_{\text {down }}^{-1}$ defined in respective intervals $U P$ and $D O W N$. Then the following assignment is valid:

$$
\begin{gathered}
l_{A}:=x_{u p}(0), \quad l_{A}^{-}:=x_{u p}(1), \\
l_{A}^{+}:=x_{\text {down }}(1), p_{A}:=x_{\text {down }}(0)
\end{gathered}
$$

If a constant function equal to 1 is added within the interval $\left[1_{A}^{-}, 1_{A}^{+}\right]$we get UP and DOWN with one interval (Fig. 2), which can be treated as a carrier. Then the membership function $\mu_{A}(x)$ of the fuzzy set defined on the $\mathrm{R}$ set is defined by the following formulas:

$$
\begin{array}{lll}
\mu_{A}(x)=0 & \text { for } & x \notin\left[l_{A}, p_{A}\right] \\
\mu_{A}(x)=x_{\text {up }}^{-1}(x) & \text { for } & x \in U P \\
\mu_{A}(x)=x_{\text {down }}^{-1}(x) & \text { for } & x \in D O W N .
\end{array}
$$

The fuzzy set defined in that way gets an additional property which is called order. Whereas the following interval is the carrier:

$$
U P \cup\left[1_{A}^{+}, 1_{A}^{-}\right] \cup D O W N
$$

The limit values for up and down parts are:

$$
\begin{aligned}
& \mu_{A}\left(l_{A}\right)=0 \\
& \mu_{A}\left(1_{A}^{-}\right)=1 \\
& \mu_{A}\left(1_{A}^{+}\right)=1 \\
& \mu_{A}\left(p_{A}\right)=0
\end{aligned}
$$

Generally, it can be assumed that ordered fuzzy numbers are of trapezoid form. Each of them can be defined using four real numbers:

$$
A=\left(l_{A}, 1_{A}^{-}, 1_{A}^{+}, p_{A}\right) .
$$

The figures below (Fig. 4) show sample ordered fuzzy numbers including their characteristic points.

Functions $f_{A}, g_{A}$ correspond to parts $u p_{A}$, down $A \subseteq R^{2}$ respectively, so that:

$$
\begin{gathered}
u p_{A}=\left(f_{A}(y), y\right): y \in[0,1] \\
\operatorname{down}_{A}=\left(g_{A}(y), y\right): y \in[0,1]
\end{gathered}
$$

The orientation corresponds to the order of graphs $f_{A}$ and $g_{A}$. The figure below (Fig. 5) shows the graphic interpretation 


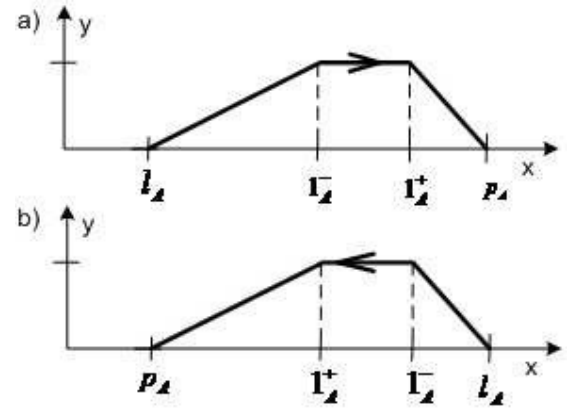

Fig. 4. Fuzzy number that is ordered a) positively b) negatively

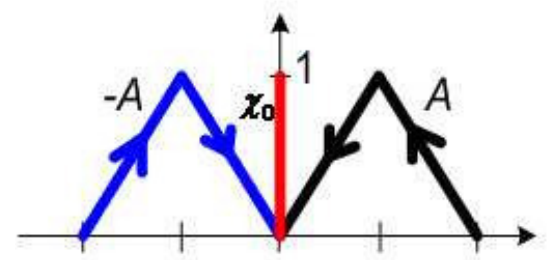

Fig. 5. Opposite numbers and the real number

of two opposite fuzzy numbers and the real number $\chi_{0}$. Opposite numbers are reversely ordered [11].

Definition 2: A membership function of an ordered fuzzy number $A$ is the function $\mu_{A}: R \rightarrow[0,1]$ defined for $x \in R$ as follows [9], [6]:

$$
\mu(x)=\left\{\begin{array}{rll}
f^{-1}(x) & \text { if } & x \in[f(0), f(1)]=\left[l_{A}, 1_{A}^{-}\right] \\
g^{-1}(x) & \text { if } & x \in[g(1), g(0)]=\left[l_{A}^{+}, p 1_{A}\right] \\
1 & \text { if } & x \in\left[l_{A}^{-}, 1_{A}^{+}\right]
\end{array}\right.
$$

The above membership function can be used in the control rules similarly to the way membership of classic fuzzy numbers is used. All quantities that can be found in the fuzzy control describe selected part of the reality. Process of determining this value is called fuzzy observation.

\section{B. Arithmetic operations in OFN}

The operation of adding two pairs of such functions is defined as the pair-wise addition of their elements, i.e., if $(f 1, g 1)$ and $(f 2, g 2)$ are two ordered fuzzy numbers, then $(f 1+f 2, g 1+g 2)$ will be just their sum. It is interesting to notice that as long as we are dealing with an ordered fuzzy number represented by pairs of affine functions of the variable $y \in[0,1]$, its so-called classical counterpart, i.e., a membership function of the variable $x$ is just of trapezoidal type. For any pair of affine functions $(f, g)$ of $y \in[0,1]$ we form a quaternion of real numbers according to the rule $[f(0), f(1), g(1), g(0)]$ which correspond to the four numbers $\left[l_{A}, 1_{A}^{-}, 1_{A}^{+}, p_{A}\right]$ as was mentioned in previous paragraph. If $(f, g)=A$ is a base pair of affine functions and $(e, h)=B$ is another pair of affine functions, then the set of typical operation will be uniquely represented by the following formulas respectively:

$$
\begin{aligned}
& \text { - addition } A+B=(f+e, g+h)=C, \\
& C \rightarrow[f(0)+e(0), f(1)+e(1), g(1)+h(1), g(0)+h(0)]
\end{aligned}
$$

- scalar multiplication $C=\lambda A=(\lambda f, \lambda g)$,

$$
C \rightarrow[\lambda f(0), \lambda f(1), \lambda g(1), \lambda g(0)]
$$

- subtraction $A-B=(f-e, g-h)=C$

$$
C \rightarrow[f(0)-e(0), f(1)-e(1), g(1)-h(1), g(0)-h(0)]
$$

- multiplication $A * B=(f * e, g * h)=C$

$$
C \rightarrow[f(0) * e(0), f(1) * e(1), g(1) * h(1), g(0) * h(0)]
$$

\section{Association of OFN order with the environmental trend}

In order to explain calculations presented in this sections, authors made the following assumptions concerning the context of changes taking place in the studied object (the impounding basin).

- close context - understood us the trend visible locally in the basin. It defines the trend of the object, i.e. if it is gradually filled or if the water level gradually falls. It is defined locally by the management of the dam,

- further (environmental) context - understood as the global trend for the observed area. It specifies trend of specific section of the river, intensity of precipitation as well as the set of other regional data which give better image of the environment. It is defined in the disaster recovery centre.

Two context types described above will be associated with the order of OFN numbers as follows. As the current water level state is reported to the disaster recovery centre, numbers that represent that state will always be oriented according to the environmental trend (reported to the centre). Whereas order of fuzzy numbers that represent changes of the water level in the impounding basin will be consistent with the local trend defined by the management of the dam. As a result, the order will be positive when the basin will be gradually filled, regardless the rate of that process. Whereas negative order can be observed when the outflow of water from the basin starts. Trend order changes themselves, as well as boundary conditions of the moment when they should occur, will make a separate process defined be the disaster recovery centre and the dam management respectively. Nothing stands in the way to ultimately use known segmentation methods for those processes and to determine the trend. Such issues are currently present in numerous publications and their detailed description is beyond the scope of this paper. Authors of that study assumed that the order equivalent to the trend changes is provided by a trusted third party and represents the expert's opinion concerning short-term weather forecast in the region important for the water level in the impounding basin as well as for the basin itself. 


\section{AN EXPERIMENTAL COMPARISON OF FUZZY NUMBERS ARITHMETIC}

\section{A. Elementary arithmetic operations}

To automate computational experiments, authors of this study developed dedicated programme Ordered FN, which also efficiently supports graphic interpretation of operations being performed on ordered fuzzy numbers. Additionally, it is equipped with the Calc L-R module.

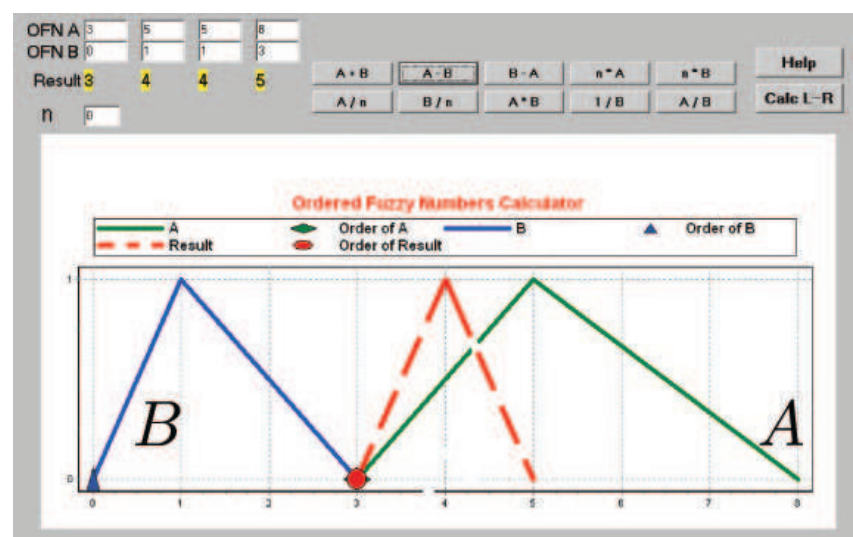

Fig. 6. Main screen of the programme

Ordered FN is equipped with an additional module which is started using the Calc L-R button. It includes procedures to calculate the sum, the difference and the product of L-R numbers. This allows to compare some results obtained from operation on L-R and OFN numbers. The introduced fuzzy number has a form of $(m, \alpha, \beta)$ where $\alpha$ and $\beta$ are left and right-side dispersions. Shown here is an example of the software application. It includes the summary of simple arithmetic operation results in OFN and L-R notation. As shown in the attached figures (Fig. 6), subtraction of 3-3 is different for L-R than for OFN numbers.

\section{B. Comparison of calculations on $\mathbf{L}-\boldsymbol{R}$ and $\boldsymbol{O F N}$ numbers}

Majority of operation performed on L-R numbers, regardless if it is addition or subtraction, increases the carrier value, i.e. the areas of non-accuracy. Hence performance of several operations on those numbers can cause such big fuzziness that the resulting quantity will be useless. It is impossible to solve an $A+X=C$ equation using L-R representation through operations because for that interpretation $X+A+(-A) \neq X$ and $X * A * A^{-1} \neq X$. Whereas every inverse operation on L-R numbers will increase the carrier. It is often impossible to solve the equations using analytical (computations) method either. However, it is possible to break the stalemate using certain empirical methods. The situation is totally different in case of operation on ordered fuzzy numbers. It is possible to solve the above mentioned equation using an analytical method.

Example 3.1: Problem: There was a rapid surge of water in the impounding basin at night. The management of the dam has to send reports to the disaster recovery centre including the value of the water level change comparing to the previous state. Unfavourable weather conditions do not allow for precise measurement.

Data:

$A(1,1,2) \quad$ - previous measurement [mln $\left.\mathrm{m}^{3}\right]$

$C(5,2,3) \quad$ - current measurement in $\left[\mathrm{mln} \mathrm{m}^{3}\right]$

Mathematic interpretation:Hence the problem comes down to determining $X$ number that satisfies the equation $A+X=$ $C$ as $A(1,1,2)+X=C(5,2,3)$

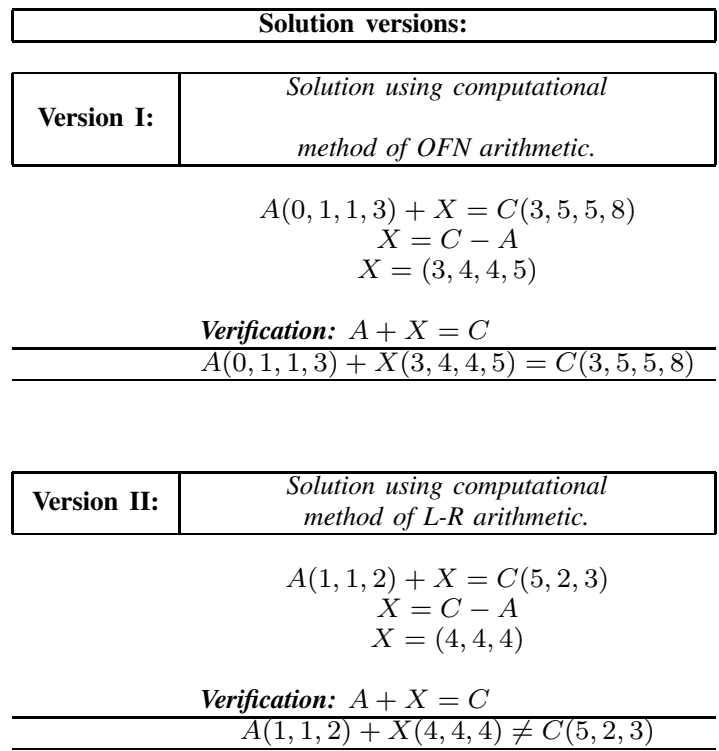

As a result of operations on L-R numbers we obtain the outcome $(4,4,4)$. However, the verification through addition $(A+X)$ gives the result different from $C$. Correct result $(4,1,1)$ can only be achieved using empirical method. It is problematic and not always feasible.

\section{CONCLUSION}

Operations performed on ordered fuzzy numbers are often more accurate than operations performed on classic fuzzy numbers. Results of operations performed on them are the same as those obtained from operations on real numbers. Performing multiple not necessarily causes large increase of the carrier. The situation is different for L-R fuzzy numbers, where several operations often lead to numbers of high fuzziness. An infinitesimal carrier is interpreted as a real number and thus for OFN numbers one can apply commutative and associative property of multiplication over addition. The possibility to perform back inference on them allows to reproduce input data by solving an appropriate equation. This very property is an added value that makes this fuzzy logic extension worth to promulgate. Calculations performed on ordered fuzzy numbers are easy and accurate. It is worth mention the multiplication here, where the same procedure is used for all ordered fuzzy numbers regardless their sign. Whereas multiplication of L-R numbers is different for two positive numbers than for two negative ones. Another completely different procedure is used 
for multiplication of numbers of indefinite signs and for fuzzy zeros. It also seems to be very interesting to associate OFN numbers with trend of changes taking place for studied part of the reality. We are convinced that new applications of this property of OFN, shown here in the example of the fuzzy observation of the impounding basin in unfavourable weather conditions, will be introduced with the passing of time. Hence it seems that introduction of OFN gives new possibilities for designers of highly dynamic systems. With this approach it is possible to define trend of changes, which gives new possibilities for the development of fuzzy control and it charts new ways of research in the fuzzy logic discipline. Broadening it by the theory of ordered fuzzy numbers seems to allow for more efficient use of imprecise operations. Simple algorithmization of ordered fuzzy numbers allows to use them in a new control model. It also inspires researchers to search for new solutions. Authors did not use defuzzyfication operators [12] in this paper, which in themselves are interesting subject of many researches. They will also contribute to development of the comparative calculator created here. Although authors of this study are not so enthusiastic like the creators of OFN as regards excellent prospects of this new fuzzy logic idea, but they are impressed by possibilities for arithmetic operations performed using this notation. Even sceptics who treat OFN with reserve as it is generalization of fuzzy logic, can benefit from this arithmetic. After all OFN can be treated as internal representation of fuzzy numbers (heedless of it's authors' intention). With this new kind of notation for fuzzy numbers and fuzzy control, it is possible to achieve clear and easily interpreted calculation, which can be arithmetically verified regardless of the input data type. Perhaps the OFN idea will become another paradigm of fuzzy logic, just like the object oriented programming paradigm has become dominant in software engineering after the structured programming paradigm. Whichever scenario wins, at least some aspects of OFN arithmetic seem to be hard to ignore a priori.

\section{REFERENCES}

[1] L. Zadeh, "Fuzzy sets," Information and Control, vol. 8, no. 3, pp. 338 $-353,1965$.

[2] J. Łukasiewicz, O logice trójwartościowej, 1988.

[3] D. Dubois and H. Prade, "Operations on fuzzy numbers," International Journal of systems science, vol. 9, no. 6, pp. 613-626, 1978.
[4] — , "Fuzzy elements in a fuzzy set," in Proc. IFSA, vol. 5, 2005, pp. $55-60$.

[5] _ "Gradual elements in a fuzzy set," Soft Computing, vol. 12, no. 2, pp. 165-175, 2008.

[6] W. Kosiński, "On fuzzy number calculus," Int. J. Appl. Math. Comput. Sci, vol. 16, no. 1, pp. 51-57, 2006.

[7] W. Kosiński, P. Prokopowicz, and D. Ślęzak, "On algebraic operations on fuzzy numbers," in Intelligent Information Processing and Web Mining. Springer, 2003, pp. 353-362.

[8] W. Kosiński and D. SŞysz, "Fuzzy numbers and their quotient space with algebraic operations,” Bull. Polish Acad. Sci.Ser. Tech. Sci., vol. 41, pp. 285-295, 1993.

[9] W. Kosiński, P. Prokopowicz, and D. Ślęzak, "Ordered fuzzy numbers," Bulletin of the Polish Academy of Sciences, Ser. Sci. Math, vol. 51, no. 3, pp. 327-338, 2003.

[10] W. Kosiński, P. Prokopowicz, and K. Frischmuth, On Algebra of Ordered Fuzzy Numbers, Soft Computing Foundations and Theoretical Aspects, J. K. Krassimir T. Atanassow, Olgierd Hryniewicz, Ed. EXIT, 2004.

[11] P. Piotr, "Algorytmization of operations on fuzzy numbers and its applications (in polish)," Ph.D. dissertation, 2005.

[12] T. Bednarek, W. Kosiński, and K. Węgrzyn-Wolska, "On orientation sensitive defuzzification functionals," in Artificial Intelligence and Soft Computing. Springer, 2014, pp. 653-664.

[13] G. Gerla, "Fuzzy logic programming and fuzzy control," Studia Logica, vol. 79, no. 2, pp. 231-254, 2005.

[14] S. Gottwald, "Mathematical aspects of fuzzy sets and fuzzy logic: Some reflections after 40 years," Fuzzy sets and systems, vol. 156, no. 3, pp. 357-364, 2005.

[15] C. L. Walker and E. A. Walker, "The algebra of fuzzy truth values," Fuzzy Sets and Systems, vol. 149, no. 2, pp. 309-347, 2005.

[16] I. Couso and S. Montes, "An axiomatic definition of fuzzy divergence measures," International Journal of Uncertainty, Fuzziness and Knowledge-Based Systems, vol. 16, no. 01, pp. 1-17, 2008.

[17] J. Dombi, "Towards a general class of operators for fuzzy systems," Fuzzy Systems, IEEE Transactions on, vol. 16, no. 2, pp. 477-484, 2008.

[18] L. A. Zadeh, "Is there a need for fuzzy logic?" Information sciences, vol. 178, no. 13, pp. 2751-2779, 2008.

[19] A. Grabowski, "On the computer certification of fuzzy numbers," in Proceedings of the 2013 Federated Conference on Computer Science and Information Systems, M. P. M. Ganzha, L. Maciaszek, Ed. IEEE, 2013, pp. 51-54.

[20] I. Bošnjak, R. Madarász, and G. Vojvodić, "Algebras of fuzzy sets," Fuzzy Sets and Systems, vol. 160, no. 20, pp. 2979-2988, 2009.

[21] Z. Xu, S. Shang, W. Qian, and W. Shu, "A method for fuzzy risk analysis based on the new similarity of trapezoidal fuzzy numbers," Expert Systems with Applications, vol. 37, no. 3, pp. 1920-1927, 2010.

[22] T. Nawarycz, K. Pytel, M. Gazicki-Lipman, W. Drygas, and L. Ostrowska-Nawarycz, "A fuzzy logic approach to the evaluation of health risks associated with obesity," in Proceedings of the 2013 Federated Conference on Computer Science and Information Systems, M. P. M. Ganzha, L. Maciaszek, Ed. IEEE, 2013, pp. 231-234.

[23] B. Rębiasz, B. Gawet, and I. Skalna, "Fuzzy multi-attribute evaluation of investments," in Proceedings of the 2013 Federated Conference on Computer Science and Information Systems, M. P. M. Ganzha, L. Maciaszek, Ed. IEEE, 2013, pp. 977-980. 